\title{
The Digital Culture within Enterprises and Public Administration: Legal Aspects and Repercussions on the Country's Socioeconomic Fabric
}

\author{
Ida D'Ambrosio
}

Additional information is available at the end of the chapter

http://dx.doi.org/10.5772/intechopen.77606

\begin{abstract}
In line with the international framework, Italy is going through a process of transformation and innovation affecting the services provided to citizens and enterprises; this process aims at administrative simplification through the use of digital technologies.

Digitization is affecting the Public Administration significantly. The unprecedented and large-scale impact of new technologies is resulting in a more effective system and, above all, in a closer distance between Public Administration and users (both citizens and enterprises). The final aim is to facilitate the access to services and the relaunch of the economy.

Italy is at a disadvantage in comparison with other countries.
\end{abstract}

Keywords: digitalization, innovation, industrial revolution, enterprises, public administration

\section{Introduction}

A new and exciting challenge humanity is called to face today is the so-called "fourth industrial revolution," since we are facing a true transformation of the way we live, work, and relate to others.

We are witnessing deep change within several production sectors, characterized by the inception of new enterprise models and by the call into question or the reconsideration of the present production, consumption, transportation, and shipping sectors. Regarding the social context, there is a paradigm shift in place that is affecting the way we work and communicate 
but also the way in which we access information, express ourselves, or spend our free time. Meanwhile, governments and institutions alike are object of reorganization, as well as the educational systems, social services, and the transportation sector. The technological innovations that are useful in order to change practices, production, and consumption systems also offer the opportunity to promote the regeneration and conservation of the environment, avoiding, this way, the hidden costs originating from the outsourcing of such processes.

The changes can be defined as historical if we consider their intensity, reach, and pace. Unlike the previous industrial revolutions, which have taken place at a linear velocity, the present one is happening at a staggering one. This is based on the heterogeneous nature of the world we live in, which is constantly interconnected, and in the fact that the existing technologies create some new and better performing ones.

The transformation has its roots in the digital revolution, and it combines several types of technologies, originating unprecedented paradigm changes both at an individual level and in financial, business, and social terms.

The transformation involves entire systems, countries, enterprises, sectors, and companies in general.

The first industrial revolution has affected the years between about 1760 and 1840 and has introduced the first mechanical production, also favored by the creation of the railway system and by the invention of the steam engine.

The second industrial revolution, started at the end of the nineteenth century and ended at the beginning of the twentieth century, has implemented mass production thanks to the advent of electricity and to the introduction of the assembly line.

The third industrial revolution started at the beginning of the 1970s, and it is often referred to as the "digital revolution" or "information technology revolution," since it was determined by the development of semiconductors, which were high-level computing devices, of personal computers and by the diffusion of the Internet.

As mentioned earlier, nowadays we are witnessing the beginning of a fourth industrial revolution that, started in the twentieth century, is a direct consequence of the digital one. It is characterized by a more spread usage of the Internet, which can be accessed with growing frequency through mobile devices that keep getting smaller but more powerful and inexpensive, and by the recurring employment of artificial intelligence and of automatic learning tools [1].

The digital technologies based on hardware, software, and network devices do not represent a novelty, but, unlike what used to happen in the third industrial revolution, they stand out for a higher level of integration and sophistication, contributing to the transformation of society and global economies.

In Germany, the expression "Industry 4.1" was introduced for the first time at the Hannover Fair to describe, at global level, how the industrial revolution would change the configuration of the so-called "value chain," that is, the strategically relevant business activities.

By favoring the creation of "smart factories," the industrial revolution in place creates a work environment in which the physical and virtual production systems interact in a flexible 
fashion, enabling the personalization of the products and the realization of new operational models.

However, it is to be noted that the fourth industrial revolution is not just about the possibility to have available permanently connected "intelligent" systems and devices. Its scope is certainly more ample and it also includes the simultaneous development of many innovations in a wide range of fields [2].

Innovation travels at an unprecedented pace, both in terms of development and in terms of diffusion.

What is amazing is not only the pace of the change but also the efficiency of scale deriving from it. Digitalization means automation, which in turn is translated in higher or otherwise significant efficiencies of scale. Today, it is possible to create value thanks to the employment of a number of workers that is definitely lower compared to 10 or 15 years ago, because digital companies have marginal costs almost amounting to zero. Furthermore, in the digital era, new companies supply and commercialize information; therefore, the stock maintenance expenses, transport, and product duplication expenses are nearly null [3]. Some companies that create breakthrough technologies seem to need very little capital in order to function successfully.

Aside from the diffusion rate and ability, the fourth industrial revolution is unique, considering the growing harmonization and integration of different disciplines and innovations.

\section{The digital skills level required and the supply system}

Digitalization and its related enabling technologies (Cloud [4], mobile computing, cybersecurity [5], big data [6], social, Internet of Things (IoT) offer great opportunities to companies, but they also demand cultural changes and a deep renovation of the areas of expertise. In this scenario, both private companies and public entities are involved in pinpointing and introducing new digital competences within the organization and to launch training activities for the reskilling of the existing staff members. This demand for new skills involves an adaptation both in the offer system, in terms of expressed skills, and in the training system. As observed by the Osservatorio delle Competenze Digitali 2017 (Digital Skills Monitoring Unit) [7], the needs that generate a demand for new skills within private companies are mostly new and innovative production methods (for $51.1 \%$ of companies), offer innovation (46.7\%) and employment of new tools and digital channels in customer relations (40\%) [8]. Concerning the innovations in production, the paradigm Industry 4.0 entails the introduction, in the plants, of detectors, smart controllers, 3D printers, connected machinery, business intelligence and analytics software, and other digital technologies aiming to make production more efficient, in integrated mode, remote, and in real-time logic. New competences will be needed for the architecture design and for the creation of applications for the development of new products and services within the factory. The new professions will be needed in order to promote and launch in the best way the transformation of the manufacturing industry: the Industry 4.0 concept does not just deal with adopting new technologies, but it involves new governance and company organization models, as well as new professional 
figures. In public administration entities, the demand for new competences is guided by $360^{\circ}$ innovation and digitalization of the administrations, which involves both the services to citizens and the services to enterprises (for $85.7 \%$ of the entities) and the internal workflows and processes $(64.3 \%)$.

All these factors have an impact also in the information and communications technology (ICT) sector, which will in turn have to adjust the competences so as to better support the offer innovation $(58.1 \%)$, the digital strategies to relate to clients $(48.6 \%)$, and the multichannel system (41\%). In this context, both technological and soft skills are essential in order to align business strategies and technological evolution to support innovation and improve internal procedures. The technological trends, which impact several levels of the demand for new digital competences, add to the needs pinpointed by companies, public entities, and ICT suppliers. The main technological trend is cloud, indicated by $71.4 \%$ of ICT suppliers and by $55.4 \%$ of the user companies, whereas big data, Internet of Things, mobile and app economy, and cybersecurity follow [8]. The most critical profiles that are more difficult to find on the market are: for the cloud area: cloud computing strategist, cloud security architect, and cloud computing engineering; for the big data area: big data specialist, big data scientist, big data architect, and big data software engineering. The Internet of Things will keep being one of the main drivers of the digital transformation of companies and it will require professionals with skills in architecture design and development and innovative applications, in managing and monitoring of services (meeting the service level agreement (SLA)/key performance indicator (KPI), internal client support, and system governance requirements), and in issue management and resolution. This is the context in which are emerging the IoT consultant and IoT engineering profiles, which are crucial to designing and implementing new processes and new smart products. They are accompanied by the IoT security software engineer, for systems protection, and the architecture mobile and IoT solutions engineer, for innovative processes management and development of related products. Cybersecurity, as well as other technological paradigms stands across the digital transformation processes, representing an essential element: the progressive and growing digitalization of the companies' internal and external activities in all market sectors is determining, indeed, an increase in cyber threats. In this context, the crucial profiles for companies are the cybersecurity consultant, the cybersecurity architect, and the cybersecurity project manager.

Finally, one key issue is the training of the new figures, which does not always keep pace with the demand. The master's degree programs in emerging digital areas (e.g., big data) in Italy are still very few. One risk is that the gap on the new areas will remain and will be accompanied by an excess in other areas linked to traditional technologies, limiting the digital transformation plans. Digital transformation is quickly changing the features of the skills needed by companies, public administrations, and citizens in order to keep pace with globalization, but the lack of awareness might leave behind many unaware small and medium-sized enterprises (SMEs). Because of this, there is a lack of a "modernized" job market, lack of a training system that is able to keep pace with the skills required, and, finally, a lack of awareness especially in small entrepreneurs regarding the transformations in place and the urgency to acquire all the opportunities they offer rather than being overwhelmed by them. 
The main subject is that of digital competences, which are today's strategic asset meant to enable all the potentialities of digital transformation.

From the analysis of the job offers via web, there is one data that stands out: the gap between what companies look for and the professional knowledge of those seeking an occupation.

This is so important that if it keeps running this way, the educational system is bound to only prepare students for unemployment. The job offers related to new and emerging digital professions have grown by 280\% from February 2013 to April 2017.

However, regarding job offers that refer to positions that are not strictly technological, as already mentioned, the component related to digital skills is growing, especially in the human resources (HR), accounting, and marketing sector.

The transformations in place are outlining more and more the need to exit the traditional evaluation framework and select "digital" professional figures in order to capture the liquid world of cross-disciplinary skills, among which shines the ability to capture and manage the continuous change: it does not make sense anymore to follow the trend of a "genetic" research of new professions. What is needed is to capture inclinations, versatility, and ability to cooperate, conjugated with unique abilities to "live" the new technology rather than just use them.

In the Italian manufacturing fabric, one of the main criticalities is cultural and linked to small businesses: many companies cannot decode the new needed skills, many others would not have the financial resources to attract them and employ them anyway. However, most importantly, an entrepreneurial awareness of the strategic edge of the digital factor is still missing.

\section{Advantages and disadvantages of digitalization}

By now, digitalization is the key to competitiveness: it represents an essential leverage in order to increase the ability of enterprises to play a growing role in the international markets and the ways to realize this are many. The use of the new technologies in the management of a company, as a matter of fact, does not just apply to the communication and marketing areas but also to production, sales, business model, learning modalities, customer interaction, market access, and activities.

With digitalization, the production method changes, as well as the way the enterprise trades and communicates. Furthermore, digital technology, aside from simplifying our daily life, has generated new possibilities for development for all those enterprises that are able to keep pace with change and that know how to take advantage of the new opportunities that digital technology offers, resulting in the fact that nowadays more entities decide to evolve so as not to be excluded and so as to have the possibility to emerge in the market.

Digital transformation in general is financially sustainable for the SMEs and entails several advantages such as: saving on turnover, simplification of the access to information, improvement in business processes, and enhancement of competitive advantage. Furthermore, there is a strict connection between digitalization and internationalization: as a matter of fact, from 
a research conducted by DOXA on behalf of Google, it appears that enterprises that have used more digital instruments (such as software and the Internet) for their business, at the same time, have seen their export sales volumes soar.

Despite many enterprises in Europe have been rapidly digitalized so as to be more competitive on a global level, the more traditional sectors of production and services such as the construction industry, the agro-food sector, the textile sector, and the iron and steel industry have only marginally benefitted from the opportunities offered by digitalization and are therefore still behind in digital transformation compared to their competitors in other European countries. In order to fill the gap, several European Union (EU) member states have approved strategies to sustain industry digitalization; however, it seems necessary to sustain a more global approach on a European level so as to avoid market fragmentation and to benefit from the digital evolution. Italy, for example, is among the member states that are less digitally developed: infrastructures and competences, indeed, are below the European average, whereas Spain is among the countries in their "catching-up" phase, which, despite remaining below the European Union average, are advancing faster than other member states and are therefore considered as recovering. Delaying the digitalization process of an enterprise means to limit its business tools and not allow it to exploit all the opportunities offered by the new technologies.

Digital transformation is an articulate process which requires management skills, but it is not just an innovative work. This transformation entails cost and process management benefits: more efficiency, more efficacy, as well as a range of definitely better services to offer to customers and suppliers. It is therefore necessary for Italian and Spanish enterprises to convert to this transformation quickly and efficiently, since only through digitalization, it is possible to think about the future of the entrepreneurial fabric.

There are numerous studies that have hypothesized the advantages of the digital industrial revolution and also many that have highlighted the possible disadvantages of it. Among several doubts, though, such as the jobs that could be lost, there are also some encouraging evaluations.

The last study written by the Capgemini Digital Transformation Institute states that the investments in smart factories could generate a $27 \%$ increase in production efficiency during the next 5 years, entailing an added value of $€ 500$ billion to the global economy. An encouraging factor is that the evaluations are made by enterprises that are active within the manufacturing sector. In addition, according to the survey, most of the enterprises interviewed seem to have understood that rather than losing jobs, the best strategy with the advent of automation is to enhance the skills of their employees, reducing inefficiency. It is not a case that $54 \%$ of the interviewed have declared to have inserted their employees in digital training courses, whereas $44 \%$ of them are investing in new workforce already trained. In our country, according to a research conducted by Ranstad in 33 countries, two workers over three deem necessary an increase in digital competences, since, according to $70 \%$ of the interviewed, to date, Italian companies do not have available the digital skills needed in order to start a digitalization process. After all, more digitalization would guarantee a positive impact on the companies' competitiveness, both in terms of internationalization and in terms of occupation and production. We live in a country with a strong motivation toward digital economy but 
that struggles to create the necessary skills to support it: there is a lack of long-term strategies which involve companies and training systems, there is a lack of a general view meant to coordinate the courses of digital transformation, there is a lack of resources to make the public administrations (PA) adaptable to change.

The demand for ICT professions is constantly growing. Every year it increases by $26 \%$ on average, with peaks of $90 \%$ for new professions linked to digital transformation such as business analyst and big data specialists, just to highlight the evolution toward "data-driven company." The demand for new digital professions increases by $56 \%$ in total: cloud specialists, cybersecurity, IoT, service development, service strategy, robotics, cognitive and artificial intelligence. There is definitely more demand in the Northwest (48\%).

Regarding the traditional ICT profession, the demand for software analysts is in constant rise ( $+24 \%$ in the last year). Regarding salary, ICT pays: in sector companies, wages in 2016 have increased with peaks of $+5.7 \%$ for employees and $+4.9 \%$ for management.

The jobs are there but many positions remain empty. The estimate is that in the 3 years between 2016 and 2018, there could be 85,000 new jobs requiring ICT qualifications within a total occupation rate which could rise by $3.5 \%$ per year by 2018 to reach 642,000 units. For these positions, the market requires $62 \%$ college graduates and $38 \%$ secondary school graduates, but our training system offers too many secondary school graduates (8400 in excess) and too few college graduates in ICT courses (4400 in deficiency). The good news is that the number of enrollments in ICT college courses grows every year, however, the dropout rate is very high (60\%), especially in the 3-year information technology degrees.

The competences linked to big data, data science, cybersecurity are slowly making their way in university courses while cloud keeps being neglected. In non-ICT faculties, the digital skills are overlooked and there is no training related to them for about a half of the 4362 existing degree courses. However, the cooperation among schools, universities, enterprises, and associations are increasing, albeit slowly. It is definitely a strategic area that needs to be amplified by overcoming the issues linked to regulatory framework dispersion, to organizational coordination, and to access to incentives. The demand for new digital profiles, from the point of view of enterprises and public administration, has been analyzed by a qualitative panel and has highlighted the growing demand for new profiles linked to processes, products, and strategy innovation in a digital framework. The profiles that are crucial to the ICT chain are: business analyst, project manager, and security analyst. The gap can be filled by reconverting the existing resources through classroom training (which is increasing among young people) and digital learning. External research uses, as preferred channels, the interaction with technical and scientific faculties (47.6\%), the personal/professional network (47.6\%), and social media $(42.9 \%)$.

In user companies, the common most important profiles to be found are: information systems manager, ICT security manager, and project manager.

What is critical today is the skill digital rate, that is, the grade of pervasiveness of the digital competences within a single profile requested by the market: according to the analysis of the web vacancies in 2016, in ICT professions, it affects for about $68 \%$, with peaks of $80 \%$ for new 
business figures related to the IoT, mobile, and cloud area, whereas, in other professions, the incidence is growing and linked to both changes in the areas of automation in processes that are stimulated by Industry $4.0(63.6 \%)$ and in the digital relation with the customer in service and business areas $(54.6 \%)$.

In total, $85 \%$ of the interviewed PAs, instead, need digital skills in order to face the digitalization of services to citizens and enterprises, linked, for example, to SPID, PagoPA, and Fascicolo Sanitario Elettronico (Electronic Health Dossier). Unfortunately, it is difficult to find these skills externally because of the ongoing hiring freeze, and it is also difficult to train existing resources because of the difficulties in diverting them from their core activities.

But if we look at the jobs for the future, the scenario changes. The new professions will be called change manager, agile coach, technology innovation manager, chief digital officer, IT process and tools architect and will be formed by an articulate mix of skills meant to strategically manage the change imposed by the big data, cloud, mobile, social, IoT, and security areas. The new figures will be especially composed by technological, managerial, and soft skills such as leadership, emotional intelligence, creative thought, and change management.

\section{Digitalization and innovation in public administration}

In line with the international framework, Italy is undergoing a process of transformation and innovation of the services to citizens and enterprises in a view of simplification, also through the use of digital technologies and of cooperation among all operators of the system.

The explosive and transversal effect of the new technologies will lead not only to a more efficient system but most of all to a shortening of the distance between public administration and users (either citizens or enterprises), to ease the access to service and to relaunch the economy, particularly in some strategic productive sectors such as tourism, culture, and agriculture.

In this context, the Italian government is at the forefront to reach the country's digital growth objectives and to accompany public administrations and users in a journey toward modernization and transformation.

The 2014-2020 Strategy for Digital Growth document defines precisely the planning for the transformation of the country. Consistently with this strategy, the Italian government has identified the main objectives, such as to guarantee to citizens and enterprises a secure and protected access to digital services, to centralize the civil registries, to develop the electronic payment systems in Pas, and to have enabling platforms for the provision of digital services to citizens in a mobile first framework.

The organizations that have reached the highest degree of digital development have users as their primary interest.

Italy also recognizes that if citizens and enterprises are put first, digital innovation will become a public investment tool with an immediate impact on the improvement of the services already provided as well as the improvement of the quality of life. 
The lack of digital skills represents one of the main obstacles to digital transformation. Italy is aware that these profiles are crucial and also it is aware of the importance of the diffusion of a digital mind-set to the purpose of success in the realization of the digital strategy. One direct expression of such commitment is represented by the reorganization of the Agency for Digital Italy, which will lead to the development and to the implementation of digital skills meant to respond in an efficient way to its mission.

On an international level, the situation that emerges seems to be even more undeveloped on the procurement side, which is spoiled by an excessive level of regulation and the lack of flexibility. The redesign of the Italian procurement model is certainly at the center of a government strategy which implies the simplification of the procedures and the identification of agile solutions that will be able to support all levels of public administrations, like, for example, the pre-commercial procurement.

In order to really transform the public sector by taking advantage of digital technologies, the public administrations will have to face a real journey, a "digital journey."

Governments and administrations find themselves in very different phases of this "journey." While a small percentage might be considered as going through a "phase of maturation," governments and administrations, for the most part, are still in the beginning or developing phases of the digital course. What differentiates the leaders of digital technology from the others is a clear strategy for digital technology, as well as a leadership and a culture that are ready to sustain transformation. This should not surprise, as the history of technological progress abounds with examples of companies that have focused on technology without investing in the organizing skills that are needed to guarantee its impact.

Public administrations have shown to be particularly prone to fall in this trap. Particularly, the first attempts to introduce computers in schools have not determined an improvement in performance, since they were not accompanied by essential modifications of the teaching methods that would allow them to be adjusted to the new technology.

The failure to employ corporate resources within public entities is a classic example of unmet expectations due to the incapability to modify processes or to develop change-favoring cultures.

The digital development of an organization is influenced for the most part by its digital strategy. The "lack of a strategy" is the main barrier that prevents organizations in their beginning phase to fully take advantage of the digital trends.

However, it does not seem to be among the five main barriers for entities in a phase of digital development.

As organizations go from the initial phase to the more advanced one, the problem represented by the lack of strategy seems to withdraw and the barriers move toward the portfolio management, financing, and data and systems security management. The organizations that find themselves in a developmental phase have a clear strategy that moves toward a radical transformation. The potential impact of a digital strategy is broadly determined by the purpose and the reach of it. Also, the importance that organizations ascribe to the use of 
digital technology in order to improve the innovation degree and the decisional process varies according to the level of digital development reached.

In the same way, the public sector managers expect that the human resources sector adjust to digital solutions and accept them in order to face the challenges linked to personnel management.

The unrestrained changes that stimulate digital transformation question consolidated leadership and governance models. Before digital technology, the new projects could be evaluated through deep analysis, the investment decisions could be based on cost/efficiency ratios, and the final objective of most plans consisted of a fixed target.

In the new digital era, leaders are asked to make decision more rapidly in the face of a constant evolution of the possible scenarios.

In public entities, the whole world of hierarchies and the government structures are often firmer and more marked than in the private sector. Government entities in a phase of digital development are characterized by a culture that favors digital transformation: they promote innovation, favor collaborative work environments, and adopt a positive attitude toward risk propensity. Furthermore, not always innovation and collaboration are the byproduct of digital technology, rather they can be promoted toward management methods that favor digital technology.

Risk propensity is becoming a cultural norm for organizations undergoing a phase of digital development.

Public entities in a phase of digital development are more capable of taking risks, that is, more prone to experiment agile approaches, such as: "make a mistake in a hurry, fix a mistake in a hurry." They are five times more prepared to modify their attitude toward risks taking compared to entities undergoing an initial transformation phase.

Furthermore, the organizations that are only beginning their transformation allude to "lack of entrepreneurial spirit" and "willingness to take risks" among the most crucial barriers to the exploitation of the digital trends. Innovation and collaboration abound in those organizations undergoing a phase of digital development.

The entities undergoing a phase of digital development find themselves in a better position to benefit from collaboration. Almost all of the entities in this phase (98\%) declare that digital technologies and digital skills allow employees to work better together.

Public entities in a phase of digital development face a remarkable change in their innovative and collaborative culture determined by the digital trends: they have a probability to notice an improvement in their innovative and collaborative culture that is three times higher than what entities in their initial phases are likely to experience.

Digital culture is weaker in the defense, energy, and justice sectors. These are sectors that focus on public services that have a crucial importance, characterized by a very low errortolerance, and in which very small operational faults could have long-term negative consequences. These sectors are less willing to experiment new approaches and are the slowest 
when it comes to innovating and collaborating using new digital technologies, which could derive from the fact that they have favored risk-averse cultures, which make it more difficult to pursue innovation and experimentation.

Personnel digital development is weak in some sectors providing professional services, particularly the higher education, health, and social service sectors. These are sectors in which the service provision to the public is assigned to professionals with a proper training, who base their activity on their own judgment and who operate with a certain level of autonomy. These sectors are slower to invest in the personnel skills that are needed in order to implement a digital strategy, maybe because those are areas have always given more importance to professional judgment, subject knowledge, and interpersonal skills rather than to organizational, digital, or transformational skills. The user's attention levels are higher in sectors in which the public service is represented by the provision of knowledge to clients/citizens. This is particularly true in the information technology (IT), education, and higher education sectors. The data are not surprising; it is indeed very likely that the requests from these users evolve and change frequently, which makes the ability to put the user's needs first a crucial factor for the entities operating in such sectors.

The main barriers preventing digital transformation are: insufficient financing and too many competing priorities.

Many public entities are already facing difficulties financing basic services to citizens, not to mention investing in digital initiatives, despite digital transformation is largely recognized as a way to reach a substantial cost reduction.

The managers who attempt to stimulate change always face competing priorities; particularly, many struggle to find a balance between transformation and "guarantee a, however, low level of service." Public entities that manage to successfully pursue their objectives tend to have strategies that are realistic and coherent and very well understood business cases.

Aside from insufficient financing and too many competing priorities, the organizations that are in a developmental phase indicate security as an important barrier. For the entities in their initial transformation phase, the absence of a general strategy-together with a poor understanding of the new tendencies of the digital world - represents a considerable barrier.

The public sector has the need to enhance the digital skills of both the employees appointed with realizing the digital transformation and those in charge of guiding it.

Employing people who have the skills needed or properly train the existing employees may naturally contribute to fill the specific ability gaps. It is essential for the people directly involved in the digital transformation activity to work side by side with the recruiting specialists in order to guarantee that the acquired digital skills be the right ones.

Digital transformation is a phenomenon that goes farther beyond technologic implementation. As a matter of fact, it requires the ability to look at "old" problems and processes with new eyes. To have a digital mentality means to have an approach toward reality that is different from the one that is adopted by most organizations, especially in the public sector. It implies a different way to consider stakeholders, a different way to launch products 
and services, and a different way to work. There is no shared definition of what makes a digital mentality, but the organizations that "become" digital tend to have some peculiarities in common: open functionalities, cocreation, special attention to users and clients, and an agile work method. Such peculiarities are not yet rooted in most public administration entities.

The management of the cultural aspect is a challenge for the organizations that face digital transformation. The existing cultural limits often put to test the attempts to integrate the foundations of the digital world (attention to the user, open functionalities, and agile development) within public entities.

Most public organization face cultural issues linked to the migration toward digital culture.

For the provision of digital services, most public entities open source technology in moderation.

Public entities might not even need of an internal development team, but it is essential that they can count on procurement specialists, who must be able to effectively monitor the work carried out by the suppliers. Procurement and commercial strategies must change in a radical way in order to allow digital transformation.

The main changes that public entities want in the procurement process are: agile development, less strict terms and conditions, and a more decentralized model. Any modification of this process should consider such aspects.

The approaches of the public sector to procurement are conceived to guarantee that the public funds be spent efficiently, but the risks of a lack of public investments in the digital era are different than what they were before.

The cause of this is the growing speed of the change itself. Historically, the change was slower; it was based on assumptions that entailed that the work modalities would keep steady. The procurement routine within the whole public sector expected the provision of services at the lowest possible cost according to the set model. This favored the drafting of long-term supply contracts and low unit costs, as well as having as a side effect to freeze the business models to the moment of the process in which the organization invested in the IT sector, chose its suppliers, and defined its processes.

In the digital era, there is nothing less desirable than this. It is an era of unrestraint changes, in which 5-year-old business models are made rapidly obsolete by smartphones, big data, connected detectors, and by the innovations of the near future. The public procurement system must change in order to incentivize innovation and flexibility.

\section{Digitalization in enterprises}

Digitalize an enterprise means to adopt an economic and social thought system that is completely different, that starts from the structure of the company itself. The modification of the enterprise crosses three main structures and they are:: business model; 
- production; and;

- activity.

To make sure that digitalization proves really effective, it is necessary to involve these three dimensions in a logics and information exchange project between the three areas themselves. Digitalization of business models means sharing information regarding products and services offered by an enterprise. The aim of the digitalization of business models is essentially to increase the company's know-how through data sharing and offering additional services related to the products sold.

The development of machinery that is technologically more efficient and effective, as well as their introduction in enterprises will allow them to grow by $25 \%$, awarding them the possibility to face a rising and constantly evolving demand. Furthermore, the variety of machinery and equipment introduced will give the possibility to meet the needs of both the big enterprises and the smaller companies because of their applicability in both mass and niche production. Finally, the digitalization of the activities implies the development of digital knowledge, which may allow an enterprise to have a greater information flow than the other parties involved in the production chain and, consequently, a better resource management. The interweaving among various sectors contributes to make the enterprise agile and to make it more competitive, since it allows it to better understand the market requests. The activities mentioned above aim to introduce a series of innovations within companies that, in a market that is characterized by the domination of technology, they will not be able to overlook. The Industry 4.0 phenomenon articulates itself in processes that involve all the phases of the activities of the enterprise. The partner occupying the most relevant position in the digital innovation is SAP, a completely new management software that is used to lessen the bureaucratic-administrative load of many company operations, as well as to manage the stock in the best way.

Another aspect that is considered to be very important in the digitalization of enterprises is the positioning, also called searching engine optimization (SEO).The creation of online contents that are interesting and attractive for customers is an essential pathway toward the reciprocal exchange of information and the creation of a collaborative relationship outside the enterprise. The evolution of the Internet, in this respect, is useful to the creation of promotional campaigns, to the improvement of link building, and to practice online marketing activities, that are very useful in order to face an ever-changing and evolving market.

\section{Digitalization and regulatory framework}

In the past few years, public administrations have had to face a real digital revolution. In the same way, companies need to digitalize their processes. Truthfully, Article 40 of the Digital Administration Code has required public administrations to create their original documents electronically for quite a while. Such evidence has been reclaimed and confirmed by the legislator in 2016. It defined that public administrations create their original documents, including those related to bars, public registries, and lists by means of information technology. The 
essential lack of enacting technical specifications, which should have described in detail the process of digitalization including the formats, the descriptions of the processes, and the roles and duties of the responsible professional figures involved, has hindered the change to a PA that would be fully digital in its processes [9-11].

Electronic invoicing started on June 6, 2014 for central entities to continue on to all the others, including local ones, on March 31, 2015. The process continued with the procedures computerization plan through SPID and with other regulatory obligations such as the PagoPA adjustment.

Within this framework, the eIDAS regulation on the identification and fiduciary services came into force on July 1, 2016, and it will impact electronic signature and certified delivery services. With the advent of this new regulation, solutions such as SPID will be enhanced and even the PEC service will have to be modified if it is to have a European role aside from the nationwide one.

Focus on processes is the common denominator for the technical specifications mentioned and for all the digitalization requirements of implementing legislation within the PA: Public administrations and companies alike will need to implement completely digital processes and new responsibilities, which will have to be assigned to well-defined professional figures and will have to account for the digital storage of their own deeds and electronic documents. In this framework, the document management and storage manuals are clearly identifiable as process documentation, whereas the document management manager and the document storage manager are to be considered as process managers.

On the basis of what has been discussed so far, it could be said that a correct digitalization must take into account the following three factors:

1. a deep process review and a correct documentation and identification of the figures involved;

2. a proper use of the various technologies with a profound knowledge of the technical and regulatory implications of their use; and

3. an approach focused on the users in which the PA is to be considered as a unique service providing office implementing in its back end all the internal and external PA services required by the regulations and by the need to digitally interact with citizens.

In order to meet this objective, both public administrations and companies must not look to a technological or application solution; rather they must retain a governance strategy made of competences and training, so as to guarantee a digitalization of the processes that today are managed with analogue tools and methods. It is not possible to think that what is done with paper must be done using digital tools and electronic documents: what needs to be done is a complete redesign and a methodological reconsideration of the processes in the moment they are switched from analogue to digital.

Therefore, the approach methodology toward this digital evolution is important because of the concept itself of document in a silent public administration, since an electronic document 
is no longer a digital inversion of an analogue document, but it becomes a completely new digital object made of information, data, records, and integrated information systems.

If we look at the most complete possible document, we realize that much information is outside this container, this file, and often this information is probably very important or it adequately reassumes the whole content and therefore the whole information or practice that is contained within the document. Even if the document is protected by the law, by a regulation (like a document registered by a public administration), there is always a part of information that does not remain within the registered document but hovers in its surrounding: in the information system used, in our documents related to that registered document, probably in a PEC, or on the net or on cloud. This shows an emerging clear problem: all the information to be managed are so many that it is not possible to confine them within only one electronic document, whether it is saved, registered, or managed according to the present regulations.

Any technical specification, standard, and regulation would not be able to confine adequately all the information that should be saved in time, in order to make them recoverable outside the external support and format.

Therefore, digitalizing and dematerializing the processes before the documents, saving the information without thinking about the document anymore, becomes essential.

\section{Conclusions}

The drive toward innovation involves all the most important sectors of the economy, with a forecast of annual average increase of $2.6 \%$ for the 3 years between 2017 and 2019. The realization of the big projects of the PA runs low.

In 2016, the digital market in Italy (information technology, telecommunication, and contents) has grown by $1.8 \%$, reaching $€ 66,100$ million. In the first quarter of the last year, the growth has had an acceleration, hitting $2.8 \%$ compared to the same period the year before, confirming the growth provisions for the 3 years between 2017 and 2019. In a context that confirms a renewed vitality, thanks to the leading role of the most innovative components and the country's general rise in awareness regarding the potentiality of the digital culture, some criticalities persist and they need a solution. The first one concerns the pace of the recovery that, however quite good, is still not enough to address the delay and to involve so many SMEs that animate our productive fabric. The second one concerns the lack of digital specialists, which is likely to have an influence on the companies' investments and on the effect of the innovation encouragement policies.

The Italian digital market is moving again, and it bodes constantly improving growth rates at least until 2019, on the lead of the digital transformation processes in all main sectors. The average yearly growth rates forecasted between 2016 and 2019 are $4.4 \%$ every year in the industry sector, $4 \%$ in the banking sector, $4.5 \%$ in the utility sector, $4.2 \%$ in the insurance sector, $3.6 \%$ in the transportation sector, and $4.7 \%$ in the great distribution. These are very encouraging dynamics that, however, find less ground in the PA sector, in which the digital expense is expected to lower, with a yearly growth rate of a little less than $2 \%$, with the 
exception of the health sector, which seems to forecast an average yearly growth rate of $3 \%$ circa [8]. As for territory, the polarization toward the northwest, which contributes to $38.3 \%$ of the total digital expense, with a growth rate of $2.3 \%$ in 2016 , is set to rise.

The subject of digital skills has a strategic scope. For the 3 years between 2016 and 2018, it has been forecasted that there will be a need for 85,000 new specialists, 65,000 of which are going to be first employment subjects and more than a half of them are going to have a college degree and will be able to cover needs that will be possible to satisfy only in part. There already are very strong criticalities for the data scientist, business analyst, project manager, and security analyst profiles and for many others that are needed for the digital transformation projects.

Concerning the strategy for the country's digital growth, there are important progresses in place, particularly in the PagoPA system (with 15,601 Pas adhering and 11,332 active) and in the PA's electronic invoicing, that are now widespread, and in the European best practice. There is still a lot to be done in order to accelerate the diffusion of SPID (with growing services but on a base of only 1.5 million digital identities issued) and of the National Population Registry.

Another promising work in progress is the program Industry 4.0, which subsidizes with hyper- and super-amortizations the digital and system components of the new industrial automation and that has already started to considerably affect a market that by the end of 2016 had reached $€ 1831$ million(+18.2\%).

By 2019, the forecasts announce a progressive enhancement of the overall market growth rate, given the progressive recovery dynamics in place for the telecommunication services (TLC) and for software, solutions, and ICT services.

\section{Author details}

Ida D'Ambrosio

Address all correspondence to: i.dambrosio@unifortunato.eu

Economic Law, Benevento, Italy

\section{References}

[1] Klaus Schwab, The fourth industrial revolution, 2016, by Franco Angeli s.r.l., Milano, p. 20

[2] Klaus Schwab, The fourth industrial revolution, 2016, by Franco Angeli s.r.l., Milano, p. 21 
[3] Klaus Schwab, The fourth industrial revolution, 2016, by Franco Angeli s.r.l., Milano, p. 24

[4] P. Mell, T. Grance, The NIST Definition of Cloud Computing. NIST, Special Publication 800-145, September 2011

[5] G. M. Schneider, J. L. Gersting, An introduction to computer science, Italian edition, Apogeo, Milano 2007

[6] A. De Mauro, M. Greco e M. Grimaldi, A Formal definition of Big Data based on its essential features, in Library Review, vol. 65, n³, 2016, pp. 122-135

[7] NetConsulting cube, 2017

[8] Cfr. La domanda di competenze digitali e il sistema di offerta, in Il digitale in Italia 2017, in Rapporto Assinform Information Technology in Italia. (The demand for digital competences and the offer system, in The digital culture in Italy 2017, in Assinform Information Technology Report in Italy)

[9] DPCM 3/12/2013 For Document Retention (abrogating a previous CNIPA regulation dated 2004)

[10] DPCM 13/11/2014 On Document Preparation

[11] DPCM 21/03/2013 which pinpoints typical unique analogue document types for which, for reasons of public law, remains the obligation to preserve the original analogue document 
